\title{
UTILIZAÇÃO DE TELEMETRIA PARA DIAGNOSTICO AUTOMOTIVO À DISTÂNCIA
}

\author{
Fernando C.R.Teixeira ${ }^{1}$, Diego Riquero Tournier ${ }^{1.2}$ \\ ${ }^{1 e} 1.2$ Robert Bosch Ltda. \\ E-mails: fernando.teixeira@br.bosch.com, diego.riquero@br.bosch.com
}

\section{RESUMO}

O presente trabalho tem como objetivo apresentar os mais novos desenvolvimentos em sistema de diagnostico veicular a distancia via tecnologia de internet móvel. Este trabalho caracterizou-se inicialmente pelo estudo das tecnologias disponíveis para monitoramento e diagnóstico veicular, como o padrão OBD (On-board Diagnostics), e os protocolos usados para comunicação das diversas ECU (Engine Control Unit) presentes no automóvel. Também são estudada as tecnologias de conexão a internet móvel.

\section{INTRODUÇÃO}

O controle das funções de veículos para diagnosticar, em tempo real, problemas no motor pode representar um atividade importante, especialmente em veículos usados para transporte de cargas. Este controle permite diagnosticar ou prevenir problemas graves de forma antecipada. Atualmente, este controle é feito por meio dos sistemas OBD. Tais sistemas são utilizados na maioria dos automóveis leves e pesados que circulem atualmente nas estradas.

Este tipo de cotrole data dos anos 1970 e início dos anos 1980, quando os fabricantes começaram a utilizar meios eletrônicos para controle de funções do automóvel e diagnosticar problemas no motor em tempo real, motivados também, para atender aos padrões de emissão de poluentes da agência U.S. Environmental Protection Agency (EPA).

O OBD-II (ou OBD2) é o padrão mais recente da indústria automotiva que foi introduzido nos modelos de veículos a partir de 1996, fornecendo o controle do motor quase por completo, também monitorando as partes do chassi e dispositivos acessórios, e a rede de controle de diagnóstico do veiculo.

Dada a importância do monitoramento, do diagnóstico e da evolução dos sistemas ao longo do tempo, os fabricante de veículos e sistemas automotivos estão cada vez mais investindo no desenvolvimento de sistemas (software e hardware) capazes de acessar a rede CAN do veiculo, com o objetivo de enviar dados da rede para um servidor utilizando a infraestrutura da rede de telefonia Móvel GSM/ GPRS. O objetivo principal desta interface é permitir que o usuário ou o reparador acessasse parâmetros operacionais do veiculo remotamente.

O objetivo principal deste trabalho é estudar as tecnologias disponíveis para monitoramento e diagnóstico veicular, como o padrão OBD (On-board Diagnostics), e os protocolos usados para comunicação das diversas ECU (Engine Control Unit) presentes no automóvel. Também são estudadas as tecnologias de conexão a internet móvel. 
Para atender ao objetivo este artigo está dividido em cinco tópicos: introdução; a revisão bibliográfica com as questões teóricas que sustentam o desenvolvimento deste estudo; a proposta do sistema de transmissão; a análise e a discussão dos resultados e finalmente as conclusões.

\section{FUNDAMENTAÇÃO TEÓRICA}

\subsection{Telemetria}

A telemetria surgiu no ano de 1845 com o desenvolvimento do primeiro sistema de transmissão de dados entre o Winter Palace e o quartel da armada russa [1]. Em 1874 foi desenvolvida uma rede de sensores atmosféricos em Mont Blanc, França, com transmissão de dados em tempo real para Paris. Mais tarde, em 1906 foram montadas umas séries de estações sísmicas na Rússia, com transmissão de dados para o Pulkovo Observatory [1].

Um sistema de Telemetria consiste na medição de dados de um analisador e envio desses dados para um acumulador para posterior análise. Esta é uma tecnologia muito empregada em diversas áreas da ciência, com a medicina [2] e [3], a biologia [4], e até mesmos parques temáticos [5], e a aplicação mais conhecida da industrial automotiva é o automobilismo desportivo F1.

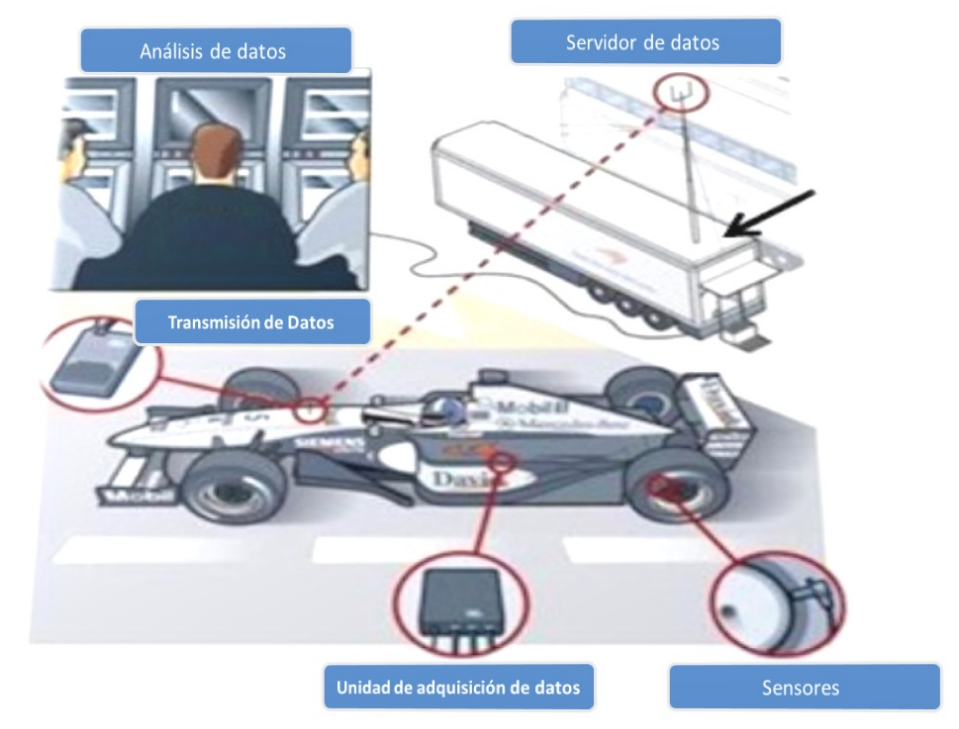

Figura 1. Configuração básica de um sistema de telemetria aplicado a F1

\subsubsection{Telemetria utilizando Telefonia Celular}

Sistemas de Telemetria via Internet móvel já existem há tempos, conforme apresenta [6]. Ao movimentar-se, um dispositivo celular troca a central de comunicações com a qual mantém conexão constantemente, mudando assim seu endereçamento, tornando este sistema de comunicação bastante confiável. 
Através das redes de dados GPRS é possível realizar diversas aplicações e promover acesso a qualquer equipamento remoto. Assim é possível obter informações e controlar equipamentos em qualquer lugar que possua infraestrutura de rede de telefonia celular GPRS.

A telemetria automotiva tem a sua maior utilização em veículos de uso comercial, aonde é instalado um computador de bordo e vários sensores no veiculo, com a função de extrai informações tais como identificação do motorista, quantidade de combustível consumida, distância dirigida, local visitada, rotas utilizadas, duração das viagens, velocidade, bem como uma série de eventos customizados pelo cliente tais como freada brusca, aceleração brusca, porta aberta, direção na chuva, etc.

A vantagem deste sistema, e que as informações críticas podem ser transmitidas em tempo real para o gerente da frota, contribuindo para reduções de custo de consumo de combustível, pneus, manutenção bem como redução no número de acidentes.

A desvantagem deste sistema é o custo, por causa da grande quantidade de sensores que necessitam ser instaladas no veiculo, dependendo da qualidade da instalação, poderá influenciar no funcionamento de componentes eletrônicos do veiculo, com, por exemplo, falhas no alarme, ruído no sistema de som e fuga de corrente, ocasionando descargas eventuais da bateria reduzindo sua vida útil.

A rede de comunicação automotiva possui todos os parâmetros necessários para a execução de uma telemetria, com o beneficio de utilizar os sensores do veiculo, sem a necessidade de instalação de sensores e grandes intervenções no sistema elétrico do veiculo. No próximo item serão apresentadas as características e aplicações da rede de comunicação automotiva.

1.2. Rede CAN: Portas abertas para a integração das tecnologias de comunicação e diagnóstico

Atualmente os veículos são equipados com um grande número de unidades de controle eletrônico que, para realizar as suas funções necessitam de um intenso intercâmbio de dados e de informações.

Os métodos convencionais de comunicações de dados ponto a ponto conectados através de um fio, já atingiu as suas limitações. Por um lado, já é quase impossível lidar com a complexidade dos chicotes, o que inviabiliza e limita o desenvolvimento de unidades de controle eletrônico.

A solução para tais limitações está na empregabilidade de sistemas de barramentos seriais de dados, próprios para o uso veicular, sendo ele a rede CAN, que atualmente se tornou um padrão para a indústria automobilística. 
Existem três ares de aplicação de redes no veiculo, cada uma com seus próprios requisitos, sendo eles: rede multimídia, aplicação multiplex e Aplicação em tempo real.

Rede multimídia: Aplicações de rede para comunicações móveis conectam componentes, tais como sistema de navegação, telefone ou equipamentos de áudio e vídeo com visualizador central e unidades operacionais como, por exemplo, um computador de bordo. Nesta aplicação, para proteger outros sistemas de rede do veículo contra acesso não autorizado e interferências externas como, sistema de som, canal móvel, internet, os domínios de redes são separados por restrições de acesso (Firewall).

Aplicação multiplex: As aplicações do tipo multiplex são adequadas para controle e regulagem de componentes eletrônicos na área de carroceria e conforto, como por exemplo, controle da climatização, controle de travas central e posição dos bancos. As taxas típicas de transmissão de dados estão entre as velocidades de 1kbit/s e $125 \mathrm{kbit} / \mathrm{s}$ [7]

Para reduzir os custos nesta área de aplicação foram desenvolvidos vários caminhos alternativos, como por exemplo, conexões econômicas ponto a ponto que podem ser empregadas entre o alternador e controle eletrônico do motor e redes locais, com taxa de transferências de 20kbit/s, podendo ser instaladas até mesmo nas portas dos veículos.

Aplicação em tempo real: As aplicações em tempo real interligam redes de sistemas eletrônicos, como gerenciamento de motor, troca de marchas e programa eletrônico de estabilidade que serve para controlar a tração e o movimento do veiculo. As taxas de transmissão desta rede ficam entre $125 \mathrm{kbit} / \mathrm{s}$ e $1 \mathrm{Mbit} / \mathrm{s}$ para garantir a operação em tempo real [8].

Para integrar e aperfeiçoar todos estes sistemas faz - se necessário à utilização de um sistema de barramento de dados para garantia da confiabilidade e velocidade da informação trafegando na rede veicular. Para este fim, o sistema de barramento de dados CAN, é utilizado como padrão para a aplicação veicular.

O sistema de barramento de dados CAN se tornou padrão para a aplicação veicular. As unidades de controle eletrônico dos diversos sistemas do veículo não são mais integradas através de um número enorme de cabos individuais, mais sim conectadas em rede por meio de um barramento de dados. Isso elimina a multiplicidade de conexões elétricas e resultam em redução de falhas.

O protocolo CAN trabalha de acordo com o princípio de conexão multi-master [9], no qual várias unidades de controle eletrônico de mesmo nível de prioridade são interconectadas através de uma estrutura linear. A vantagem deste tipo de princípio multi-master está no fato de uma falha em algum dos integrantes da rede não impede o acesso aos demais. Em comparação com outras redes, a probabilidade de falha total é reduzida. Na rede circular ou estrela, a falha de um dos integrantes ou na unidade central é suficiente para causar falha geral do sistema. 
O CAN utiliza o endereçamento baseado na mensagem. Para isso, um identificador (Cabeçalho ou endereço) fixo é designado para cada mensagem. O identificador caracteriza o conteúdo da mensagem, como por exemplo, rotação do motor. Cada estação ou ECU (Unidade de controle eletrônico) processa exclusivamente as mensagens, os identificadores constam na sua lista de aceitação (Filtro de mensagens). Assim, o CAN não requer o endereçamento de estações para transmissão de dados e as estações não são envolvidas na configuração do sistema, facilitando o controle das diversas versões do equipamento.

O protocolo CAN é baseado em dois estados lógicos: Os bits são ou recessivos (nível lógico 1), ou dominantes (Nível lógico 0). Se um bit dominante é enviado por pelo menos uma estação, os demais bits recessivos enviados simultaneamente pelas outras estações são sobrescritos [9].

O identificador define ao mesmo tempo o conteúdo dos dados e a prioridade da mensagem. Um identificador correspondente a um número binário de baixo nível possui alta prioridade e vice-versa [9].

Quando o barramento está desocupado, qualquer estação pode começar a transmitir suas mensagens. Se várias estações começam a transmitir simultaneamente começará a haver conflitos de acesso ao barramento. Para solucionar este problema o sistema usa um sistema de arbitragem. A mensagem com maior prioridade recebe acesso primeiro sem perda de bit ou atraso. Todo transmissor que perde a arbitragem é comutado automaticamente para o receptor e repete a sua tentativa de transmissão até que o barramento esteja livre. A figura 2 apresenta o formato da mensagem CAN:

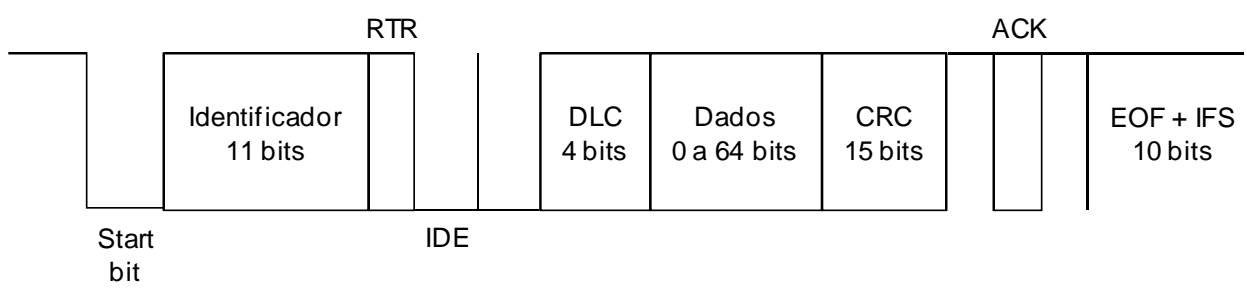

Figura 2Formato da mensagem CAN.

Como visto, o CAN suporta dois formatos diferentes de registro, diferenciados somente pelo cumprimento do identificador (ID). O comprimento do ID é de 11 bit no formato padrão e de 29 bit no formato expandido. Portanto, o registro transmitido tem no máximo 130 bit (Formato padrão) ou 130 bit (Formato expandido) [9]. Isso assegura que o tempo de espera até a próxima transmissão (que pode ser urgente) seja o mínimo possível. O registro de dados é composto de sete campos consecutivos:

- O início do registro indica o começa da mensagem e sincroniza todas as estações.

- O campo de arbitragem é composto pelo identificador da mensagem e bits adicionais de controle. Durante a transmissão deste campo, o emissor verifica a cada bit, se ele ainda detém prioridade ou se uma estação com maior prioridade esta 
transmitindo. $\mathrm{O}$ bit de controle determina se a mensagem é classificada como registro de dados ou registro remoto.

- O campo de dados dispõe de um conjunto de informação entre 0 e 8 bytes. Uma mensagem de comprimento 0 pode ser usada para sincronização dos processos distribuídos.

- O campo CRC (Cyclic Redundancy Check) contém uma palavra para verificar possíveis interferências na transmissão.

- O campo ACK (Acknology) contém o sinal de confirmação de todos os receptores, indicando que a mensagem foi recebida sem erro.

- O fim do registro marca o final da mensagem.

\subsection{Diagnóstico na palma da mão}

Seja com um telefone celular, tablet ou dispositivo móvel com base em arquiteturas eletrônicos que usam sistemas de transmissão de dados CAN (controller Area Network), é possível fazer um diagnóstico de unidades de controle eletrônico instalado em um veículo, este é o proposta de montadoras e sistemas automotivos; e não é futurologia, veículos de produção existente para a venda no mercado com este conceito, o caso da Opel Astra OTC recentemente lançado na Europa com a opção para obter acesso a unidades de controle eletrônico do veículo via telemetria.

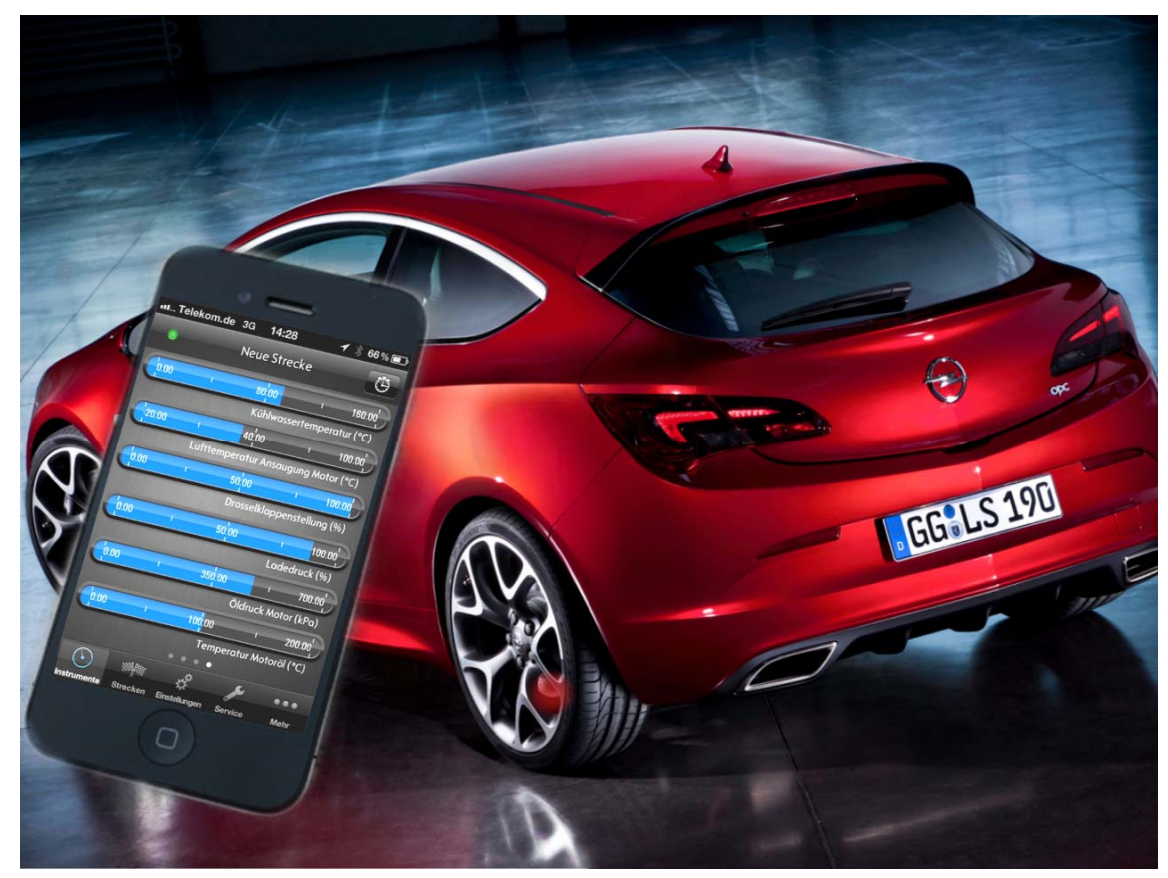

Figura 3 Opel Astra OPC com sistema de telemetria integrado.

Usando redes de comunicações móveis (GPRS), e com base nas plataformas eletrônicas CAN instaladas nos veículos, o sistema mantém todas as partes comunicadas (carro, proprietário do carro, serviços de oficina, fabricante, e outros serviços, tais como controle de tráfego, ou sistemas de emergência). 
A conectividade com o veículo é realizada por unidades de controle eletrônico que funcionam como interface de (unidade de controle do veículo), estes transmitem dados através de redes de comunicações móveis (GPRS), que, posteriormente, chegam a um servidor central de dados.

Esta comunicação é bidirecional que permite o envio e recebimento de dados. O sistema é complementado por estações de apoio e análise de dados (no caso de diagnósticos, intervenções de assistência técnica automotiva no veículo), utilizar adicionalmente portal Web permite o acesso externo e comunicação com o sistema, eventualmente, o modelo de negócios de preferência adotada pelos fabricantes é o uso de lojas virtuais para venda ou distribuição de aplicações (Apps), que será responsável pelo carregamento de dados e atualizações de sistema, substituindo o que hoje conhecemos como atualização de software de scanner.

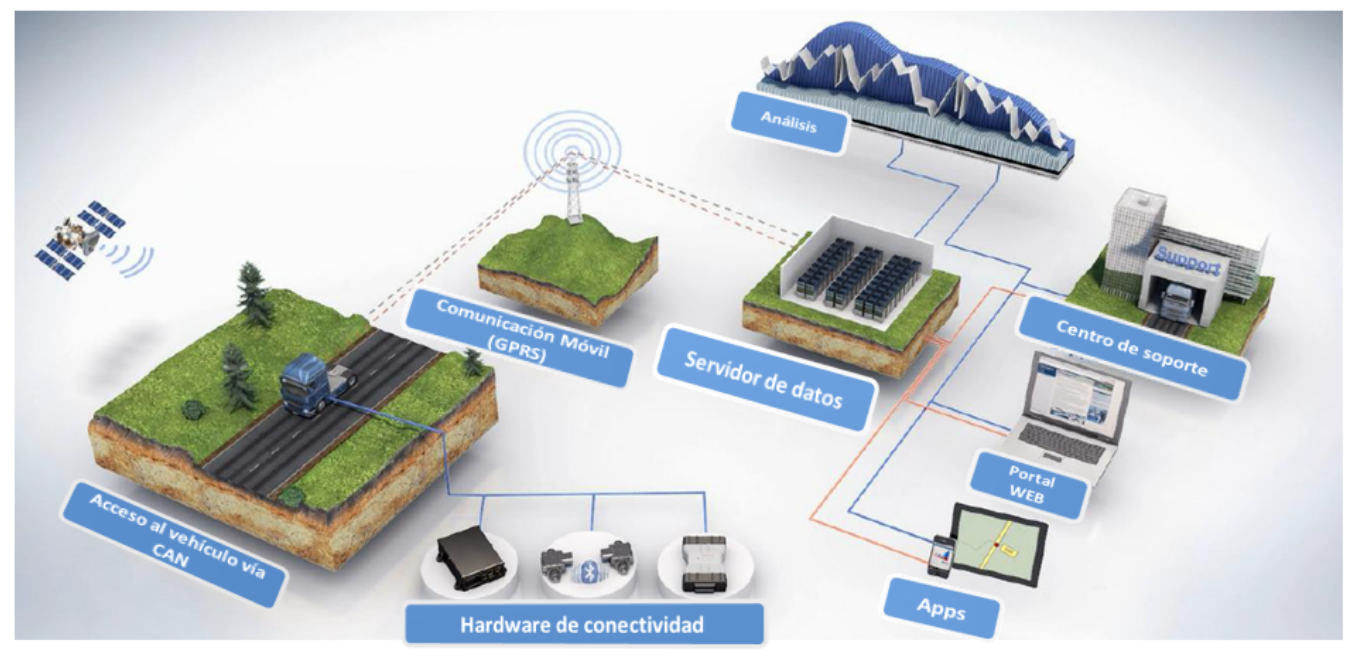

Figura 4 Sistema de telemetria aplicada a sistemas automotivos.

\section{CONCLUSÃO}

Com a inovação da tecnologia cada vez mais, a ideia de implantar um sistema de diagnostico remoto torna-se um estudo de viabilidade, pois permite uma interação maior dos usuários com os concessionários trazendo como benefícios conforto e principalmente a segurança, além de introduzir novas ferramentas nos automóveis. E que impacto tem esta tecnologia no setor de reparação automotiva? Esta é sempre uma preocupação quando confrontados com algo novo, da mesma forma que o scanner veio para revolucionar o mercado automotivo, a telemetria vem para facilitar a comunicação de grandes empresas com seus clientes, e do lado do mecânico automotivo, é essencial ser atualizado com estas tecnologias (isto não é novo), mas uma coisa que é certa, é que a telemetria não vai substituir a função do mecânico, e os veículos continuam com as mesmas características, têm componentes mecânicos controlados por sistemas eletrônicos. Isto significa que o conhecimento técnico do reparador ainda se mantém como fator de crucial no diagnostico automotivo. 


\section{REFERÊNCIAS}

[1] QUEIRÓS, J. M. R. Sistema de Sensorização e Telemetria de um VEC (Veículo Eléctrico de Competição). 2011. 117 f. Dissertação (Mestrado Integrado em Engenharia Electrotécnica e de Computadores Major Automação) - Faculdade de Engenharia da Universidade do Porto. Cidade do Porto. 2011.

[2] Gutierrez, E. M. (2006). Telemetria: Aplicação de rede de sensores biomédicos sem fio. Brasília: Master's thesis UnB - Universidade de Brasília.

[3] Paim, F. C. (2005). Desenvolvimento de um sistema de telemetria para aquisição de sinais fisiológicos com aplicação em programas de reabilitação cardíaca. Santa Catarina: Master's thesis, Universidade Federal de Santa Catarina - UFSC.

[4] Mantovani, J. E., \& Santos, J. E. (2001). Telemetria convencional e via satélite na determinação da área de vida de três espécies de carnívoros da região nordeste do estado de são Paulo. Santa Catarina: Master's thesis, Universidade Federal de Santa Catarina - UFSC.

[5] Walker, B. (2007). Augmenting amusement rides with telemetry. Advancements in Computer Entertainment Technology , pp. 115-122.

[6] Khan, N., Saleem, Z., \& Hoong, C. C. (2007). An experiment on internet based telemetry. Proceedings of the 6th WSEAS Int. Conf. on Electronics, Hardware, Wireless and Optical Communications , pp. 68-78.

[7] ISO 15 765-2, Road Vehicles - Diagnostics on CAN - Part 2: Network Layer Services, International Organization for Standardization, 1999.

[8] ISO 11 519-2, Road Vehicles - Low Speed Serial Data Communication -Part 2: Low Speed Controller Area Network, International Organization for Standardization, 1994.

[9] BOSCH, R., Manual de Tecnologia Automotiva, 25ª ed., São Paulo: Edgard Blücher, 2005. 